Bol. Acad. peru. leng. 51.2011 (69-111)

\title{
EL PADRE NUESTRO EN LA LENGUA MOCHICA
}

\section{LA PRIÈRE DU NOTRE PÈRE DANS LA LANGUE MOCHICA}

\section{THE LORD'S PRAYER IN THE MOCHICA LANGUAGE}

\author{
José Antonio Salas García
}

\section{Resumen:}

El objetivo de este trabajo es presentar la historia de la plegaria del Padre Nuestro en la lengua mochica, la cual ha sido publicada reiteradamente en el Viejo Mundo y efectuar una traducción al castellano de esta oración. Como resultado de la traducción, mostraremos fenómenos del mochica que no fueron dados a conocer ni por Fernando de la Carrera ni por Jerónimo de Oré, porque la información gramatical fue inferida de los textos mismos.

Résumé:

L'objectif de ce papier est de présenter les détails de la prière du Notre Père dans la langue mochica, qui a été publié à plusieurs reprises en Europe. Nous voulons aussi faire une traduction castillane de cette-ci. En raison de la traduction, nous montrons certains phénomènes qui n'ont pas été libérés par Jerónimo de Oré (1607) ou Fernando de la Carrera (1644), puisque l'information grammaticale a été déduite à partir des textes eux-mêmes. 
Abstract:

The first aim of this paper is to introduce the history of the Lord's Prayer in the Mochica language,which had repeatedly been translated in the Old World. Our second purpose is to make a spanish translation of this prayer. In this new translation, we will show Mochica phenomena, which Jerónimo de Oré (1607) or Fernando de la Carrera(1644) did not deal with, because the grammatical information was inferred from the texts themselves.

Palabras clave:

Lengua mochica; traducción; textos cristianos

Mots clés:

Langue mochica; traduction; textes chrétiens

Key words:

Mochica language; translation; Christian texts

Fecha de recepción:

$29 / 05 / 2011$

Fecha de aceptación:

$30 / 06 / 2011$

\section{Introducción}

El presente es el quinto trabajo de la serie de artículos destinados al estudio y traducción de los textos del idioma mochica ${ }^{1}$, los cuales fueron editados en las obras Ritvale, Sev Manvale Pervanum de Luis Jerónimo de Oré (1607) y el Arte de la lengua yunga de Fernando de la Carrera (1644). Estos textos son de tipo religioso, por haber sido escritos por sacerdotes para adoctrinar a la población en su propia lengua. El orden en el que aparecen escritos es el mismo que el de la Doctrina Christiana de 1584, aun cuando De la Carrera añada algunos más, también de tipo religioso. En

La primera oración traducida fue "La Señal de la Cruz" en Salas 2008. En el 2009 se tradujeron "Los Sentidos Corporales", "Los Enemigos del Alma" y "El Sacramento de la Comunión”. Esta última oración aparece en la bibliografía con fecha 2007, porque la revista Allpanchis llevaba un atraso en la edición de sus números, pero fue editado después de iniciado el 2009. 
1787, el texto mochica del Padre Nuestro, correspondiente a Luis Jerónimo de Oré, fue reeditado por Lorenzo Hervás (1787: 93) en su libro Saggio Pratico delle Lingue, en el que figuran más de 300 versiones de la oración del Padre Nuestro en lenguas de todo el mundo. La trascripción de Hervás fue segmentada en cláusulas, con adaptaciones y errores. En el plano de las adaptaciones, notamos que la $\langle v\rangle$ pasa a ser $\langle u\rangle$. Así, $\langle$ Mvchef $\rangle$ de Oré pasa a ser $\langle$ Muchef $\rangle$ o $\langle v z i c a p u c\rangle$ se transforma en $\langle u z i c a p u c\rangle$. El diacrítico $\langle\sim\rangle$ que indicaba la nasalidad en Oré se grafica como $\langle n\rangle$ en Hervás. Ejemplo, 〈eyipmãg $\rangle$ deviene en $\langle$ Eyipmang $\rangle$. La $\langle c ̧\rangle$, tanto en su valor de fricativa como de africada, se convierte en $\langle z\rangle$. En el siguiente ejemplo se registran cambios tanto en el valor africado como en el fricativo: De $\langle c ̧ u \tilde{u} c u c ̧ i a s\rangle$ a $\langle z u n g c u z i a s\rangle$. La $\langle q\rangle$ se transforma en $\langle k\rangle$. Así, 〈ef quecan> pasa a ser $\langle$ Ef kecan $\rangle$. La yod $\langle y\rangle$ se convierte en $\langle i\rangle$ : De $\langle y x l l i s\rangle$ a $\langle i x l l i s\rangle$, salvo cuando está en ataque de sílaba con valor consonántico o no nuclear.

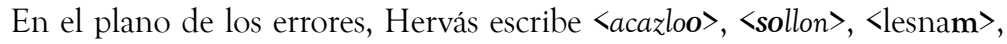
$\langle$ cuzianguic $\rangle$ (en dos ocasiones), cuando en el texto original de Oré se lee $\langle a c a z l o c\rangle,\langle x l l o n\rangle,\langle$ lesnan> y <cuçiangnic>. En las primeras décadas del siglo XIX, la versión de Hervás fue reeditada por el alemán Johann Christoph Adelung (1813: 549-550) en la segunda parte (dedicada a las lenguas de Sudamérica) del tercer volumen de su monumental obra Mithridates, que contiene -entre otras cosas- más de quinientas versiones del Padre Nuestro en idiomas del orbe entero. La reedición de Adelung no contenía traducción al alemán ni a ninguna otra lengua. Únicamente el texto en mochica tomado de Hervás.

A finales del XIX, el texto del Padre Nuestro fue trascrito por Ernst Middendorf (1892: 172) con la pronunciación que le tocó escuchar, pero tomando como base, esta vez, a Fernando de la Carrera. Middendorf pone debajo de cada palabra mochica su equivalente en alemán, pero sin ofrecer el significado de la frase, como un todo, de manera cabal. Así, traduce la flexión de segunda persona $\langle a s\rangle$ con el pronombre en alemán de segunda persona $d u$ 'tú', cuando se trata de categorías gramaticales distintas. Del mismo modo, una forma como $\langle a k\rangle$ que mantenía homonimia entre la raíz del verbo 'ver' y una conjunción relativa es traducida por Middendorf con un derivado verbal de 'ver', en vez del pronombre relativo, como hubiese sido lo correcto. Este autor 
no brinda una segmentación morfológica de cada uno de los elementos léxicos del mochica.

En 1895, con ocasión de la undécima reunión del Congreso Internacional de Americanistas, Raoul de la Grasserie (1897: 500) presentó, entre otros textos mochicas, la versión del Padre Nuestro de Luis Jerónimo de Oré y la confrontó con la versión de Fernando de la Carrera. El método de traducción es similar al de Middendorf. En ambos casos, la traducción es del mochica al francés.

En el siglo XXI, Torero (2002: 368) proporciona el texto dividido por cláusulas y segmentado morfológicamente; pero sin ofrecer el significado ni de los lexemas, ni de los morfemas. El texto del Padre Nuestro, además, se encuentra sin la traducción de cada una de las oraciones, como un todo. Simplemente, se lee el texto mochica del Padre Nuestro con la particular ortografía que Torero usaba para esa lengua, la cual difiere tanto de las fuentes existentes, como del alfabeto fonético internacional. Todo esto, segmentado por guiones para los morfemas y puntos seguidos, cuando un nombre era regido de posposición; además de los signos de puntuación convencionales para dividir las cláusulas.

En el 2004, el lingüista holandés Willem Adelaar hace un comentario de esta plegaría. Con mayor conocimiento que Middendorf acerca de gramática, Adelaar coloca el significado en inglés con información gramatical, debajo de cada palabra mochica y en una tercera línea coloca el sentido de la frase. Sin embargo, esta tercera línea corresponde a la versión estándar del Padre Nuestro en inglés, cuando el texto en mochica no, necesariamente, sigue el estándar del Padre Nuestro ni del inglés, ni del español. Por ejemplo, en un pasaje, cuya traducción literal en mochica es: "Hágase adoración a tu nombre", Adelaar (2004: 244) lo traduce por "Hallowed be thy name", usando el arcaico pronombre de segunda persona thy y la frase en pasiva be hallowed 'ser santificado', la cual no está presente en el texto mochica. Si bien, al traducir palabra por palabra, Adelaar reconoce el significado del verbo lic 'hacer', a través de make, no presenta la traducción literal en inglés, dejando -como anotamos- la versión estándar con sus variaciones de 
significado y sus arcaísmos léxicos. ¿De qué nos serviría ofrecer como traducción la versión que todo el mundo conoce del Padre Nuestro en castellano, si lo que nos interesa es la traducción literal del mochica? El sentido general de las versiones estándares está en la oración mochica, pero nuestro interés se centra más en los detalles. Ahora bien, tanto Middendorf cuanto Adelaar traducen el Padre Nuestro de la versión de 1644 de Fernando de la Carrera, quien lo titula en latín Pater Noster, tal como lo había hecho ya Luis Jerónimo de Oré. El texto que segmenta Torero también corresponde al Arte de 1644. Nosotros, por nuestra parte, proporcionaremos, adicionalmente, la traducción de Oré, correspondiente a 1607. Si bien ambas versiones (Oré y De la Carrera) son muy similares, existen pequeñas variaciones que las diferencian, tal como mostraremos en su momento. No obstante, donde creemos que radica la mayor importancia de la comparación entre los textos es en el plano de la forma, por las distintas grafías empleadas para una misma entidad léxica. La comparación sistemática de todas las palabras empleadas por Oré y De la Carrera permitirá avances en el estudio de los sonidos del mochica, a partir del análisis de sus grafías. Esa es, por cierto, una de las razones que animan esta serie de traducciones. Junto con el Padre Nuestro de Fernando de Carrera, viene un pequeño texto que explica lo que se pide en la oración y un conjunto de preguntas y respuestas, formuladas con afán didáctico. Estos últimos fragmentos no han sido materia de análisis con anterioridad, pero lo serán en el presente estudio.

\section{Corpus}

El corpus a analizar está compuesto por sendas versiones del Padre Nuestro. En la Arte de 1644, además, hay una pequeña explicación de "Lo que contiene el Paternoster" (Carrera 1644: 203) y una serie de nueve preguntas y respuestas que llevan el título de "Interlocuçion sobre el Paternoster" (1644: 203-205), lo cual está ausente en el texto de Oré, quien simplemente ofrece el Padre Nuestro, sin comentarios que lo acompañen. Como lo hiciéramos con antelación, transcribiremos el corpus tal como está en los documentos coloniales, para luego efectuar un análisis, segmentando correctamente los elementos lingüísticos y 
estableciendo una puntuación, acorde con la época actual. He aquí los textos que forman el corpus, los cuales son transcritos, tal cual aparecen en las fuentes:

\section{El Pater Noster}

(Oré 1607: 403)

Mvchef, acazloc, cuçiangnic, çũq oc licum apmucha, Piycan ñof, çũgcuçias, eyipmãg, çung, poleng munmo vzicapuc,cuçiangnic mun, Ayoyneng. ynengo, much xllon, Piycam ñof allò molun, ef quecan ñof.yxllis, acan mux efco. xllang museyo. much çiòmun, Amus tocum ñof. xllangmuse yz puçèrenic, namnum, les nan, efco, nof pissin quich. Amen Iesus.

\section{Pater Noster \\ (Carrera 1644: 202)}

Mæich ef, acaz loc cuçiang nic, tzhæng oc mang licæm mæcha, piycan nof tzhæng cuçias, eiæpmang tzhæng polæng mæn, mo æizi capæc cuçiang nic mæn. Aio ineng, inengô mæich xllon, piy can ñof allô mo lun. Efque can nof ixllæss aie aca naix efco xllãg muss eio mæich, çio mæn. Amoz tocæn ñof xllang muss emællæc zær enicnam næm lecu nan ef coñof pissin quich Amen.

Tan sólo Fernando de la Carrera (1644) escribe un pequeño texto, en el que aclara el número de pedidos que expresa el Padre Nuestro, destinando tres para Dios y el resto para el hombre:

\section{Lo que contiene el Paternoster (Carrera 1644: 203)}

ñite lecuo tem çæc e, mo Padre nuestrong nic, çopætô lecuo çio fe Dios iio pæn nopæt o quitzhieio çio fe mæich peñæss pæn.

Luego de esto, De la Carrera formula nueve interrogantes con sendas respuestas, a fin de servir como ejercicio pedagógico. Esta fue una estrategia de aprendizaje bastante empleada en el Arte de 1644, de 
manera tal que era fácil llegar a la gente por medio de diálogos sencillos que estuviesen al alcance de todos:

\section{Interlocuçion sobre el Paternoster}

(Carrera 1644: 203)

P. Ech ix temapæco, çiotem çær ær nic.

R. Temap coix ech, ech, Dios $i$ lec çiung mæich çio pij cæm næm aieçæn eix temapco çio Padre nuestrong nic, mæich ixllæss ef coçær.

P. Ech ix temapeco, Dios $i$ oc mucha ap lic ssæe?

R. Temap coix santoio çiung oc izcær xamar næm, aieçæn izcær irrim cæm næm.

P. Ech ix temapco, pijcan ñof tzhæng cuçias, çie eng læc.

R. Temapæcoix, Dios mæich nic chinæm, santoio çiung chiçærer, aieçæn eix temapco muc çæn, çiung cuçias.

(Carrera 1644: 204)

P. Ech ix temapco a me eng læc eiæp mang, tzhæng polæng mæn, mo æizi capæc, cuçiang nic mæn?

R. Temap coix, izçæc ñof nær Dios i ssap macu quem næm canang cuçiang nico angel çio mæn.

P. Ech ix tema pæco, ame eng læc aio ineng ineng ô mæich xllon piy can ñof alló mo lun?

R. Temapæcoix xllonquic, mæich ærqueng eiopæn: aieçæn peño chi çæc mæich moix eio pæn.

P. Ech ix temapco, ame eng læc efque can ñof ixllæss, aie, acan ax efco xllang muss eio, mæich, çio mæn?

R. Temapcoix, mæich, ixllæss efcoçær aieçæn eix temap $æ$ co purgatoriong ni quichò tuxllum çær.

P. Expix eng apco ame aie acan ax efco xllang muss eio, mæich, 
çiomæn?

R. Inong efcoca Dios mæich ixllæss çiema mix efco ænta, chang cædeio mæich.

(Carrera 1644: 205)

P. ech ix temapco ame eng læc amoz tocæn ñof xllang musse mæ llæc çær enic nam næm?

R. Te mapcoix Dios i lec tarro chi çær, açæn ixllinic nam ssæc, ssap er, locu er çæn æntaçæn çifa chi çær er.

P. Eño pissin quich oix eng ef quem næm, ame eng læc lecunan efco ñof pissin quich?

R. Demoniong mæcu quich ô ix tem ef quem næm, aiecæn infiernong niquich, aieçæn timæno pissin quich.

\section{Metodología}

En primera instancia, nos ocuparemos del texto del Padre Nuestro en sus dos traducciones para que nuestro análisis se beneficie de la comparación entre ambas versiones. Una vez efectuado el cotejo de ambas, comentaremos tanto lo que contiene el Paternoster como la interlocución que se hace de esta oración en el Arte de la lengua yunga de 1644. Era una práctica usual en la época que los textos aparezcan sin traducción. Haciendo uso de las obras lexicográficas del mochica y de la Doctrina Christiana de 1584, traduciremos los textos, separando las palabras con arreglo a los siguientes criterios: 1) La división entre palabras se hará teniendo en cuenta los lexemas existentes en la frase. A guisa de ejemplo: la frase nominal que aparece en Oré (1607: 403) como $<$ Mvchef $>$ compuesta por el pronombre personal en genitivo much y el nombre ef 'padre' se separará correctamente: Mvch ef "mi padre". Del mismo modo, como la división de palabras se basa en el lexema, no existirá separación en medio de palabra, de modo tal que un vocablo como <a me> 'así' (Carrera 1644: 204) tendrá que estar junto: ame. 2) Los lexemas que contengan morfología susceptible de análisis estarán segmentados por un guión medio. De esta manera, si en el lexema <ixllæss> (Carrera 1644: 202) se distingue una raíz ixll 'pecado' y un morfema de posesión æss, entonces, lo transcribiremos como ixll-æss. 
3) Los clíticos y las frases posposicionales se transcribirán con un guión bajo. Así, la frase posposicional <mæich nic> (Carrera 1644: 203) que se traduce "en nosotros" será transcrita: mæich_nic.

Ahora, la traducción se presentará en tres renglones. En el primero, irá el texto en mochica, segmentado de acuerdo con los criterios arriba esbozados. De esta forma, contaremos con versiones normalizadas que facilitarán nuestra tarea. En un segundo renglón, irá la traducción en castellano palabra por palabra. Como la base de la traducción es el lexema, una barra diagonal / dividirá los lexemas y mediante guiones se detallarán vía abreviaturas la información gramatical que está en el mochica. Por ejemplo, a nam-næm le corresponde la traducción 'caer', pero a su vez cuenta con un morfema de finalidad (ausente en español) que abreviamos como FIN. De esta manera, entre barras, pondremos en el segundo renglón: /caer-FIN/. En el tercer renglón, transcribiremos, ya sin ninguna indicación gramatical, una traducción libre al castellano, la cual procuraremos que sea lo más literal posible.

\section{Abreviaturas}

Las abreviaturas que contienen información gramatical del texto a analizar son las siguientes: $1^{\mathrm{a}}=$ primera persona, $2^{\mathrm{a}}=$ segunda persona, $3^{\mathrm{a}}=$ tercera persona, $\mathrm{AB}=$ ablativo, $\mathrm{ADV}=$ adverbializador, $\mathrm{AG}=$ agente, $\mathrm{CAUS}=$ causativo, $\mathrm{CONJ}=$ conjunción, $\mathrm{COP}=$ copulativo, DET = determinante, EU = eufónico, FIN = finalidad, FUT = futuro, $\mathrm{G}=$ genitivo, $\mathrm{GER}=$ gerundio, $\mathrm{IMP}=$ imperativo, $\mathrm{INS}=$ instrumental, $\mathrm{CO}=$ concordancia de objeto, $\mathrm{LOC}=$ locativo, $\mathrm{NEG}=$ negación, $\mathrm{NOM}=$ nominalizador, $\mathrm{OBJ}=$ objeto, $\mathrm{PA}=$ participio activo, $\mathrm{PAS}$ $=$ pasiva, $\mathrm{PL}=$ plural, $\mathrm{PS}=$ posesión, $\neg \mathrm{PS}=$ no posesión, $\mathrm{REL}=$ relativo, $\mathrm{S}=$ singular, $\mathrm{SUBJ}=$ subjuntivo. $\mathrm{El}$ signo $ø$ se interpreta como un morfema que no tiene realización fónica, pero que es relevante para el análisis. Lo que va escrito entre los paréntesis angulados 〈> refiere a las formas ortográficas. Lo que aparece escrito entre comillas simples " refiere a los significados de las palabras aisladas y lo escrito entre comillas inglesas dobles "” corresponde a los significados inferidos de los textos.

\section{Padre Nuestro}


En esta sección, analizaremos y traduciremos por separado cada versión para, posteriormente, establecer la comparación de ambos textos. Empezaremos, en mérito a su antigüedad, con la exégesis del Padre Nuestro de Luis Jerónimo de Oré.

\subsection{Texto de Luis Jerónimo de Oré}

La versión de Luis Jerónimo de Oré (1607: 403) está encabezada por el título en letras mayúsculas: EL PATER NOSTER. He aquí la versión normalizada del texto, en la que hemos separado las palabras por lexemas y los lexemas han sido analizados en morfemas:

Mvch ef-ø, ac az loc, cuçia-ø-ng_nic

1aPL-G/ padre-PS/ REL/ 2aS/ COP/ cielo־PS-G/ LOC

Nuestro padre, que estás en el cielo.

La primera palabra much es un pronombre personal en caso genitivo. El mochica carecía de pronombres posesivos. De manera que expresaba la posesión por medio de la flexión de caso de sus pronombres personales. La estructura de la frase nominal para expresar la posesión en esta lengua hacía que existiese concordancia entre el núcleo ef 'padre' y su especificador mvch 'nuestro' (o más bien 'de nosotros'). La posesión se marcaba en una relación especificador-cabeza. Por ese motivo, el nombre ef está en su forma poseída. Sucede que en el mochica los nombres tenían dos formas: Una para la posesión y otra para la no posesión. La forma no poseída del concepto 'padre' en mochica era: efquic (Carrera 1644: 194), mientras que la poseída era el ya sabido ef. Como la ausencia de la realización fónica es la que expresa la posesión, hemos puesto el signo ø como una suerte de morfema vacío. Luego viene una cláusula de relativo que es introducida por la partícula ac. Le sigue, en segundo lugar, la flexión de segunda persona az. En los textos que hemos venido traduciendo, constatamos que el mochica se comportaba como lengua de verbo segundo, de modo tal que la flexión siempre se ubicaba en el segundo constituyente. Téngase presente que esto se aplica a constituyentes y no, a palabras. La flexión az sería la cuarta palabra de la frase, pero el primer 
constituyente de la cláusula de relativo es ac. Por tanto, es correcta su ubicación tras de esta partícula de relativo. Habiéndose cumplido con que la flexión esté en el segundo constituyente, viene la raíz del verbo copulativo loc y, finalmente, el complemento circunstancial de lugar, el cual es regido por la posposición locativa nic. Esta posposición rige al nombre cuçia en caso genitivo, porque en realidad nic era, originalmente, un adverbio de lugar (Carrera 1644: 159). Por tal motivo, no asignaba caso a los nombres. Esto hacía que el nombre necesitase de una partícula que le asignase caso y, por ello, es que se empleaba el genitivo. En tanto posposición, nic era una partícula intransitiva. La mayoría de nombres en mochica terminados en vocal están en su forma no posesiva, ya que por regla general si se les adicionaba $\langle s s\rangle$, entonces, estaban en su forma posesiva (Carrera 1644: 5). Como no hay ninguna manifestación sonora para expresar la no posesión de cuçia 'cielo', empleamos el signo ø como una marca de morfema vacío.

çũq oc-ø lic-um ap mucha-ø,

2aS-G/nombre-PS/ hacer-PAS/ pues/ adoración-־PS

Hágase pues adoración a tu nombre.

La siguiente frase se inicia con el pronombre de segunda persona en genitivo, que como vimos, expresa la posesión en esta lengua, ante la falta de pronombres posesivos propiamente dichos. La manera como está escrito 〈çũq> con un diacrítico sobre la vocal es una abreviación de un sonido nasal. En realidad, la $\langle q\rangle$ final junto con dicho diacrítico representan una consonante nasal velar. Es interesante el uso de la grafía $\langle c ̧>$, pues recuerda su antiguo valor de africada. La palabra oc 'nombre' está en su forma poseída y hace concordancia del tipo especificadorcabeza con el pronombre que le antecede. Si bien la forma no poseída está ausente en el Arte de 1644, Brüning (2004: 4) ofrece la forma no poseída ákik [ókik] (alternando con la poseída åk, 'nombre') con el significado de 'santo'. Obviamente se refiere al onomástico, por la vieja costumbre hispana de poner los nombres de acuerdo con el santoral. El término mucha, como termina en vocal y, además, por encontrarse sin ningún determinante lo hemos considerado en su forma no posesiva.

Sumamente curiosa es la estructura del verbo 〈licum> que 
significa 'hacer', pero en pasiva. Lo extraño es que no hay ningún morfema que remita a persona gramatical alguna y además la flexión verbal no sólo no ocupa el segundo constituyente antes de la raíz lic, sino que está del todo ausente. Creemos ver en esta frase una suerte de elipsis, en la que se usa la partícula ilativa ap 'pues' (Middendorf 1892: 157), como elemento pragmático que licencia está ausencia.

Piy-c-an ñof, çũ g cuçia-s, dar-CO-IMP/1aPL-OBJ/2aS-G/ cielo-PS

Danos tu cielo.

Esta oración vuelve al orden canónico, en el que si la raíz está en un primer constituyente, la flexión va en el segundo. Nótese que, tras la raíz verbal, hay un morfema que hace concordancia con el objeto animado, es decir, con ñof 'nosotros'. ¿Cómo sabemos que se trata de un morfema aparte y no, de una parte inanalizable de la raíz? Pues simplemente mostrando que existe la raíz flexionada sin el morfema: Poieiñ 'doy' (Carrera 1644: 155) y con el morfema y cierta variación alomórfica de la raíz: Piiæceiñ 'doy' (Carrera 1644: 158). La vocal del morfema en cuestión solía elidirse como en el caso que comentamos. Con todo, este morfema se incorporaba a la raíz, formando un tema que aparecía en el primer constituyente sintáctico, dejando así el segundo para la flexión de imperativo $\langle-a n\rangle$. La frase que sigue es de tipo posesivo. Esta vez, el pronombre de segunda persona posesivo $\langle c ̧ \tilde{u} g\rangle$ se encuentra escrito con $\langle g\rangle$ y no con $\langle q\rangle$, como en el caso anterior, aun cuando creemos que ambas formas gráficas representaban un mismo sonido. Finalmente, al nombre cuçia 'cielo' se le adiciona una $s$ que es el morfema que expresa la forma poseída, que concuerda con su antecedente, en una relación especificador-cabeza.

ey-ip_m-ãg, çung pol-eng_mun mo vz-ø-i_capuc, cuçia-ø-ng_nic_mun, hacer-PAS-SUBJ-3aS/2aS-G/voluntad-PS/como/DET/tierraPS-G/sobre/cielo-حPS-G/LOC/ como

Hágase según tu voluntad sobre la tierra, como en el cielo.

Una frase verbal compleja inicia esta oración. La raíz <ey> es la 
forma pasiva del verbo ai 'hacer'. ¿Cómo lo sabemos? En De la Carrera (1644: 102), la forma verbal ainæm "para hacer" se pasa de activa a pasiva, dando como resultado eiepnæm "para ser hecho". Si aislamos la raíz del morfema de pasiva, entonces, encontraremos el porqué hemos

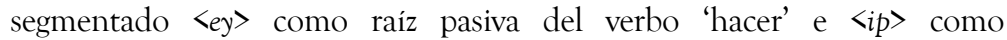
morfema de pasiva. En este caso, reconocemos que la vocal del morfema pasivo asimila el punto de articulación de la yod que está en la raíz verbal. Ahora bien, la raíz ey-y el morfema de pasiva -ip forman un tema verbal que ocupa el primer constituyente sintáctico y, por ello, la flexión <mãg> les sigue en un segundo constituyente. La flexión puede analizarse a través de dos morfemas: uno de subjuntivo y el otro de tercera persona singular. Por la manera como Oré escribió 〈eyipmãg $\rangle$ con una $\langle\tilde{a}\rangle$ con diacrítico que representaba la nasalidad de la siguiente consonante, hemos separado el subjuntivo como $\langle m\rangle$ y hemos dejado $\langle\tilde{a} g\rangle$ como persona singular. Sin embargo, la vocal $\langle a\rangle$ pertenece al morfema de subjuntivo. ¿Por qué afirmamos esto? De lo observación del mochica, hemos notado que la vocal de la flexión de persona se asimila a la de la unidad a la que se sufija o cliticiza. A guisa de ejemplo, la flexión de primera persona singular es eiñ y la de segunda, az (Carrera 1644: 30). Pues bien, compárese las formas flexionadas de primera y segunda persona de un verbo como chefnam 'tener hambre', cuya raíz termina en consonante: chefnameiñ, chefnamaz con las formas de primera y segunda persona de un verbo derivado del anterior como chefnamco 'hacer tener hambre', cuyo tema termina en vocal o: chefnamcoiñ, chefnamcoz (Carrera 1644: 66). Es fácil notar como las vocales de las flexiones se asimilan a la vocal de la base a la que se adjuntan. Lo mismo acontece con el subjuntivo. Si bien existe una flexión de tercer persona ang, esta fusiona su vocal con la del morfema de subjuntivo. Ahora, el subjuntivo del mochica, al igual que el del castellano alternaba con el imperativo para expresar órdenes. En castellano, todas las órdenes negativas se hacen con subjuntivo: "No vengas", en vez de *no ven. Los tratamientos de respeto también se hacen con subjuntivo en castellano, verbigracia, "venga usted".

Tres frases posposicionales completan la oración. La primera frase çung poleng mun "según tu voluntad" expresa con arreglo a qué se han de hacer las cosas, es decir, según la voluntad divina. El nombre poleng está 
en su forma poseída, pues la forma no poseída es polquic (Carrera 1644: 5). La frase mo vzi capuc "sobre la tierra" tiene singularidades. El nombre vz 'chacra' (Carrera 1644: 104) está en su forma poseída, la cual se manifiesta por la ausencia de morfología. De ahí el uso del signo ø. Por contraste, la forma no poseída tenía morfología: vizquic (Carrera 1644: 107). El nombre $v z$ posee un genitivo irregular $\langle i\rangle$. Este genitivo es exigido por la posposición capuc, la cual semeja la forma de nombres contraídos que están en caso locativo. En el Arte (Carrera 1644: 161), hay una lista de estas contracciones de nombres y muchas de ellas se comportaban como posposiciones que exigían caso genitivo, pues al tratarse de nombres regidos por posposiciones, entonces, no tenían la capacidad de asignar caso. Como resultado de ello, requerían el auxilio de la forma genitiva de los nombres para la asignación de caso. La última frase pospositiva cuçiang nic mun "como en el cielo" ostenta la particularidad de que una posposición rige a la otra, aunque si uno observa el español, notará que esto también se da, de modo recurrente, verbigracia: por entre las rocas, en donde la preposición por rige a entre. Por lo demás, la diferencia de significado entre las posposiciones capuc y nic nos hace pensar en que la primera denotaba lugar, pero en contacto con la superficie; mientras que la segunda era más general.

Ayo yneng. yneng-o, much xllon-ø, Piy-c-am ñof allò mo lun-ø,

DET/ día/ día-EU/ 1aPL-G/ comida-PS/ dar-CO-IMP/ 1aPL-OBJ/ $\mathrm{CONJ} / \mathrm{DET} /$ día־PS

Aquella nuestra comida del día a día, danos también este día,

La oración se inicia con una frase nominal compleja. Sintácticamente, esta frase se encuentra topicalizada. A tal punto que la flexión verbal no precede a la raíz verbal, mostrando así mayor énfasis en dicha frase nominal. El núcleo de la frase nominal es xllon 'comida' que está en su forma poseída, la cual se manifiesta por la ausencia de morfología, como lo indica el signo ø. La forma no poseída es xllonquic (Carrera 1644: 104). Tres complementos completan la frase. El primero es el pronombre personal en genitivo much, que lo encontramos en una recurrente construcción posesiva en concordancia con el nombre. El segundo complemento es una frase nominal duplicada. Sabemos que está 
regida por el núcleo, porque porta la o que indica tal rección. El hecho de que esté duplicada comunica la idea de reiteración por iconicidad. A este respecto, compárese este caso con las voces ech ech 'cosas' (Carrera 1644: 160), tuni tuni 'muchos' (Carrera 1644: 15), tzhic tzhic 'inmenso' (Carrera 1644: 195) o irr irr 'grande' (Carrera 1644: 242). En cuanto al significado de yneng. yneng, lo hemos traducido como "del día a día", usando la preposición 'de' para expresar la idea de complemento y la expresión 'día a día', porque comunica la multiplicidad de días, duplicando el nombre, tal como en mochica. El tercer complemento nominal es el determinante ayo 'aquel(la)' que suponemos cumple la función pragmática de intensificar la determinación de una frase topicalizada. La estructura de Piycam ñof ya la hemos explicado líneas arriba. Tan sólo diremos que el morfema de imperativo escrito con $\langle m\rangle$, antes que con $\left\langle_{n}\right\rangle$ parece más un error que una variación lingüística real. La partícula allò da la idea de adición y nos parece que licencia la frase nominal topicalizada, porque ocupa el sitio que le correspondería, normalmente, al objeto si no estuviera topicalizado y, además, porque vincula semánticamente a la frase nominal topicalizada con el complemento circunstancial de tiempo mo lun "este día”. Esta última frase tiene un detalle que hay que destacar, pues hemos encontrado ejemplos de este nombre lun 'día', con la misma forma tanto para la posesión, como para la no posesión. Esto lo sabemos por el tipo de genitivo que usa. Así, tenemos lunær nico "en el día” (Carrera 1644: 201) con genitivo de no posesión y luni nico "en su día" (Carrera 1644: 208) con genitivo de posesión irregular. Así, tenemos un interesante caso de un nombre que no manifiesta fónicamente el tipo de posesión, aunque de manera abstracta sí lo hace, como lo prueba el uso de los genitivos, semánticamente, especializados o bien en la posesión -e, o bien en la no posesión -ær. De modo tal que el signo ø, en este caso, podría dividirse en un morfema vacío $\varnothing^{1}$ para la posesión y un morfema vacío $\emptyset^{2}$ para la no posesión.

ef-qu-ec-an ñof. yxll-is, perdonar-CAUS-CO-IMP/ 1aPL-OBJ/ pecado-PS

Perdónanos (nuestros) pecados.

La oración se inicia con un verbo, donde hay mucho por analizar. 
El imperativo -an está después de la raíz verbal, es decir, en segunda posición, como es lo usual. Luego reconocemos una concordancia de objeto -ec- que sería correferente con el pronombre de primera persona plural ñof. Después viene la raíz propiamente dicha efqu-, en la que notamos el fósil de un morfema causativo -qu-, el cual está presente en las derivaciones de todas las formas verbales. Las raíces de los verbos eran, mayoritariamente, monosilábicas, por lo que creemos que la segunda sílaba pertenece a tal tipo de morfema. El nombre yxllis está en su forma poseída. Se puede analizar una raíz yxll 'pecado' + un morfema de posesión -is, el cual posee una vocal que -a todas luces- asimiló la vocal palatal de la raíz nominal a la que se sufijó. Es en virtud de ese morfema de posesión que hemos puesto en la traducción el pronombre posesivo de primera persona entre paréntesis, porque en el texto mochica no había un pronombre explícito.

acan mux ef-co. xllangmu-s-e-yo. much çiò_mun, como/ 1aPL/perdonar-CAUS/ enemigo-PS-G-EU/1aPL/ eso/ como Como perdonamos nosotros de (nuestros) enemigos, como eso

La oración se inicia con la conjunción modal o comparativa acan 'como' en el primer constituyente. Esta conjunción mantiene una relación discontinua con la última frase posposicional çiò mun "como eso". Ambas expresiones manifiestan la misma comparación y de ahí, su vínculo semántico. El segundo constituyente queda reservado para la flexión $\langle m u x\rangle$, la cual parece tener una consonante nasal que es una anticipación, únicamente, de tipo gráfico; porque, por el sentido de la frase, no puede tratarse de un subjuntivo ni se relaciona con la forma de ninguna flexión conocida. Luego viene la raíz del verbo que ya analizamos en la oración anterior. Sigue el sujeto de la frase en una curiosa estructura, pues viene después de la raíz verbal. De la Carrera (1644: 95) hace un comentario sobre las flexiones y los pronombres, en el que confunde la explicación del verbo segundo (totalmente desconocido para él) con las personas gramaticales. En dicho comentario, De la Carrera restringía el uso de pronombres si la flexión era postverbal y lo atribuía a las personas gramaticales. Sea como fuere, el pronombre de primera persona plural much tiene, como complemento en genitivo poseído, al 
nombre xllangmuseyo "de (nuestros) enemigos". Quizás una traducción que se adapte más al castellano sea: "Tal como eso perdonamos nosotros de nuestros enemigos". Sin embargo, hemos preferido mantenernos en el terreno de lo literal.

Amu_s tocum ñof. xllangmu-s-e yzpu-çè-re_nic, nam-num, NEG/2aS/ dejar/ 1aPL-OBJ/ enemigo-PS-G/ habladuría-NOMPS-G/LOC/ caer-FIN

No nos dejes caer en las habladurías de (nuestros) enemigos.

Una negación da principio a esta oración. Si la negación ocupa la primera posición, se reserva la flexión para el segundo constituyente, viniendo luego la raíz verbal y un pronombre personal como objeto. Una particularidad del verbo tocum es que rige al verbo no finito namnum 'caer' que está al final de la frase. Es una constante que los verbos no finitos del mochica ocupan la última posición ${ }^{2}$. Nótese, sin embargo, que cuando un verbo no finito es regido por uno finito no es posible la traducción que expresa finalidad, como en otros contextos. Ahora, como complemento del verbo no finito hay una frase posposicional, donde lo más destacado es el nombre deverbal yzpuçèr que hemos traducido como sinónimo de mællæczær 'habladuría', que es la palabra que aparece en la versión de Fernando de la Carrera, pues yzpuçèr no aparece en ningún listado de palabras del mochica y Oré no proporcionó su significado. El término yzpuçèr es un hápax.

les-na_n, ef-co, ñof piss-ø-i_nqu_ich.

más-ADV/IMP/ librar-CAUS/ 1aPL-OBJ/ mal-7PS-G/LOC/AB

Más (bien) líbranos del mal.

Un adverbio de comparación comienza la frase. Viendo la otra versión del Padre Nuestro, podemos identificarlo con el adverbio lecuna (Carrera 1644: 110). En esta forma se reconoce el adverbializador na y el nombre lecн 'cabeza'. Obviamente, su sentido de superioridad parte

$2 \quad$ El alemán, que también es una lengua de verbo segundo posiciona sus infinitivos al final de la frase, tal como el mochica. 
de una metáfora corporal. La flexión - $n$ ocupa, en forma de clítico, la segunda posición, antecediendo al verbo y su objeto pronominal. Termina la oración una frase posposicional en donde se suceden tres casos.

\subsection{Texto de Fernando de la Carrera}

La presente plegaria se aparta de la de Oré, básicamente, en el empleo de determinadas grafías, aun cuando existen pequeñas divergencias de orden, estrictamente, lingüístico que comentaremos en su momento.

Mæich ef-ø, ac az loc cuçia-ø-ng_nic,

1aPL-G/ padre-PS/ REL/2aS/ COP/ cielo-7PS-G/LOC

Nuestro padre, que estás en el cielo.

Esta oración es casi idéntica a la de Oré. Llama la atención, sin embargo, la presencia de una nueva vocal $\langle\not x\rangle$, inexistente en Oré, y el encuentro de las vocales en $\langle$ Mæich〉. Lo que en Oré correspondía a una simple vocal. En todo caso, el entorno palatal de $\langle$ ch $\rangle$ explica la presencia de $\langle i>$.

tzhæng oc-ø ma-ng lic-æm mæcha-ø, 2aS-G/ nombre-PS/ SUBJ -3aS / hacer-PAS/ adoración־PS Hágase adoración a tu nombre

Esta oración tiene variaciones con relación a la versión de Oré. La diferencia central está en que la flexión mang está en el segundo constituyente, después de la frase nominal tzhæng oc "tu nombre" que ocupa el primer constituyente. Esto es lo esperado en una lengua de verbo segundo. Carece, además, esta versión de la partícula ilativa ap que está presente en la versión anterior. Relacionamos así la presencia de la partícula ap con el distinto comportamiento flexivo entre versiones.

piy-c-an ñof tzhæng cuçia-s, 


\section{dar-CO-IMP/ 1aPL-OBJ/ 2as-G/ cielo-PS}

Danos tu cielo

Esta oración posee el mismo significado que la versión de Oré, lo cual revela un hecho de primera importancia, pues la oración se aparta de la versión estándar del Padre Nuestro en español. Como consecuencia de esto, podemos inferir la existencia de tradiciones discursivas para los rezos y plegarias que estuvieron, efectivamente, en uso.

ei-æp_ma-ng tzhæng pol-æng_mæn, mo æiz-ø-i_capæc cuçia-ø-ng_ nic_mæn.

hacer-PAS-SUBJ-3aS/2aS-G/voluntad-PS/como/DET/tierraPS-G/sobre/cielo-7PS/LOC/ como

Hágase según tu voluntad sobre la tierra como en el cielo.

En esta oración lo que más llama la atención es la diversidad de valores que puede tomar la grafía $\langle\mathfrak{X}\rangle$ si la comparamos con las vocales equivalentes en la versión de Oré. Así, la $\langle x\rangle$ del Arte de 1644 puede ser $\langle i\rangle$ en eyipmãg, $\langle e\rangle$ en poleng o $\langle u\rangle$ en capuc, del texto de Oré. Otro detalle relevante es que esta oración también se aparta, ligeramente, de la plegaria estándar en español. El hecho de que el significado sea compartido por ambos autores nos reafirma en la existencia de tradiciones discursivas propias. Estos textos no solamente fueron un ejercicio académico, sino que reflejaban muy bien una realidad.

Aio ineng, ineng-ô mæich xllon-ø, piy-c-an ñof allô mo lun-ø.

$\mathrm{DET} /$ día/ día-EU/1 1aPL-G/ comida-PS/ dar-CO-IMP/ 1aPL-OBJ/ $\mathrm{CONJ} / \mathrm{DET} /$ día־PS

Aquella nuestra comida del día a día, danos también este día,

Esta oración difiere de la de Oré tan solamente en las grafías. No obstante, sobre lo que queremos llamar la atención es sobre el trígrafo $\langle x l l>$, el cual se aparta por completo de la ortografía castellana. Incluso antes de los textos mochicas ya se empleaba esta sucesión de grafías. Zevallos Quiñones (1943: 246) proporciona, por ejemplo, el nombre del 
topónimo Xllamnacuc que corresponde a 1589. Otros topónimos con esas grafías son Xllancup, Xllan-napo o Xllanyenec.

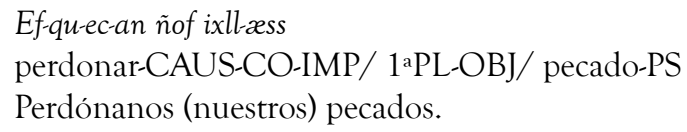

La gran diferencia con relación a la versión de Oré está en la palabra ixllæss e yxllis. La $\langle y\rangle$ es tan sólo una variación gráfica, pero la diferencia entre $\langle\mathfrak{x}\rangle$ e $\langle i\rangle$ nos parece que evidencia una distinción sonora, en la que se aprecia en un caso un fenómeno de armonía vocálica. Esto debe ser producto de la variación dialectal.

aie acan_aix ef-co xllãgmu-ss-e-io mæich, çio_mæn.

Así/como/ 1aPL/ perdonar-CAUS/ enemigo-PS-G-EU/1aPL/ eso/como Así como perdonamos nosotros de (nuestros) enemigos, como eso

Esta oración difiere de la anterior por las conjunciones empleadas y por un detalle de tipo fónico en la flexión aix, la cual típicamente es eix. Tal parece que se trataría de un caso de asimilación del timbre de la vocal anterior. Las dos palabras que forman la frase conjuntiva aie acan ocupan el primer constituyente sintáctico. Por tanto, la flexión antecede al verbo. En la versión de Oré, está ausente el adverbio aie 'así'. Nos parece que la presencia de este adverbio de modo, permite relacionar, de manera discontinua, la frase conjuntiva del inicio con la última frase posposicional çio mæn "como eso".

Amo_z tocæn ñof xllangmu-ss-e mællæc-zæ-re_nic nam-næm

NEG_2S/ dejar/1aPL-OBJ/ enemigo-PS-G/ hablar-NOM-PS-G/ $\mathrm{LOC} /$ caer-FIN

No nos dejes caer en las habladurías de (nuestros) enemigos.

La distinción central entre esta oración y la del texto de Oré estriba en el nombre deverbal mællæczær 'habladuría', que hemos tomado para interpretar la voz yzpuçèr de Oré, basándonos en todos los enormes paralelismos que existen en ambas versiones, las cuales incluso 
comparten desviaciones semánticas con relación a la versión estándar del Padre Nuestro en español.

lecu-na_n ef-co ñof piss-i_nqu_ich más-ADV/ IMP/ librar-CAUS/ 1aPL-OBJ/ mal-G/LOC/AB Más (bien) líbranos del mal.

Entre esta oración y la de Oré hay una distinción en el adverbio lecuna y lesna. Oré no hace distinción de la consonante $\langle c \mu\rangle$. Es difícil decir si estamos ante una variación dialectal o una hipodiferenciación.

\subsection{Comparación de ambos textos.}

Esta sección se dividirá en dos. Desarrollaremos, en la primera parte, las particularidades de cada una de las versiones, resaltando sus semejanzas y diferencias. En la segunda parte, presentaremos en dos columnas los signos equivalentes que compartan Luis Jerónimo de Oré (1607) y Fernando de la Carrera (1644), a fin de confrontar las grafías y términos empleados.

\subsubsection{Cotejo textual}

El resultado más destacado de la comparación de ambos textos está en los valores divergentes de las grafías empleadas por ambos autores. Sobre todo en lo que se refiere a la vocal $\langle x\rangle$, la cual tiene nueve correlatos en Oré con valor de $u$, a saber: capuc, сарæc; çũq çüg çung, tzhæng; licum, licæm; mucha, mæcha; mun, mæn; namnum, namnæm; tocum, tocæn; vzi, xizi; mvch ${ }^{\sim}$ much, mæich. Los dos últimos casos muestran que la $\langle u\rangle$ de Oré mantiene correspondencia con el encuentro vocálico $\langle æ i\rangle$. El entorno palatal de $\langle$ mæich $\rangle$ justificaría tal encuentro. Como hipótesis, Torero (2002: 322) y Hovdhaugen (2004: 14) plantearon que como las grafías $\langle c ̧\rangle$ y $\langle z\rangle$ están en contacto con $<i>$, entonces, su valor es el de una fricativa palatalizada ${ }^{3}$. Hay un caso,

$\overline{3}$ Hovdhaugen considera que $<c ̧ \sim z>$ son sonidos fricativos palatalizados, estén o 
en el que $\langle x\rangle$ tiene valor de $\langle\mathrm{e}\rangle$ : poleng, polæng. Y dos instancias, en las que $\langle x\rangle$ posee valor de $\langle i\rangle$ : eyip, eiæp; yxllis, ixllæss. Esto último se explica por un entorno palatal. También vale la pena destacar el uso de $\langle c ̧\rangle$ con el valor de africada que De la Carrera escribe como $\langle t z h\rangle$, así como la ausencia de $\langle c \mu\rangle$ en Oré, que se evidencia en la comparación entre lecuna y lesna.

En el plano léxico, Oré presenta un hápax con la palabra yzpuçèr, la cual hemos traducido gracias al texto de Fernando de la Carrera que proporciona el lexema mællæczær 'habladuría'. Por hipótesis, asumimos que ambas palabras son sinónimas, pues si uno observa los textos notará que cuando una versión mochica del Padre Nuestro se aparataba de la versión estándar, la otra también lo hacía. Esto nos lleva a pensar en una tradición oral que es registrada por los autores de los textos religiosos, a partir de experiencias reales. Es probable que De la Carrera haya tenido conocimiento de la obra de Oré, pero es poco probable que empleara un texto diferente del estándar, si es que el uso no lo hubiese justificado.

\subsubsection{Vocabularios comparados}

Hemos ordenado en columnas las palabras de Oré y De la Carrera, a efectos de comparar el vocabulario extraído del Padre Nuestro. El orden alfabético corresponde a la primera columna que trae el vocabulario de Oré. Todas las palabras han sido transcritas con minúsculas, para uniformizar la comparación. Cuando un término ha sido escrito más de una vez de distinta manera, lo hemos escrito vis-à-vis de sus variantes, separándolas mediante el signo . No obstante, cuando una voz aparece en contextos diversos con distinta morfología se ha reservado una celda aparte para su cotejo.

no en contacto con los encuentros vocálicos con $\langle i\rangle$. Torero, por su parte, analiza el valor de $\left\langle\left\langle_{c} \sim z\right\rangle\right.$ como dos fonemas distintos, de acuerdo con la presencia o ausencia de diptongos con $\langle i\rangle$. De modo tal que $\langle c ̧ i\rangle\langle c i\rangle$ o $\langle i z\rangle$ representarían fonemas diferentes a $\langle c\rangle,,\langle c\rangle,\langle z\rangle$. 


\begin{tabular}{|c|c|c|}
\hline \multicolumn{3}{|c|}{ Vocabulario } \\
\hline Oré 1607 & Carrera 1644 & Glosa \\
\hline$a c$ & $a c$ & que (relativo) \\
\hline acan & acan & como \\
\hline & aie & así \\
\hline allò & allô & también, además \\
\hline amus & amoz & $\begin{array}{l}\text { negación + flexión } 2^{a} \\
\text { singular }\end{array}$ \\
\hline$a p$ & & pues \\
\hline$a z$ & $a z$ & $2^{\mathrm{a}}$ singular \\
\hline ayo & aio & aquel(la) \\
\hline capuc & capæc & sobre \\
\hline çiò & çio & eso \\
\hline cuçiang & cuçiang & cielo (genitivo) \\
\hline cuçias & cuçias & cielo (poseído) \\
\hline$c ̧ \tilde{u} q \sim c ̧ \tilde{u} g \sim c ̧ u n g$ & tzhæng & tú (genitivo) \\
\hline ef & ef & padre (poseído) \\
\hline efco & efco & perdonar, librar \\
\hline efquecan & efquecan & perdonar (imperativo) \\
\hline eyip & eiæp & hacer (pasiva) \\
\hline ich & $i c h$ & de (ablativo) \\
\hline lesna & lecuna & más bien \\
\hline licum & licæm & hacer (pasiva) \\
\hline loc & loc & estar \\
\hline lun & $\operatorname{lun}$ & día \\
\hline$m \tilde{a} g$ & mang & $3^{\text {a } \text { singular subjuntivo }}$ \\
\hline mo & mo & este (determinante) \\
\hline mucha & mæcha & adoración \\
\hline mun & $m æ n$ & como, según \\
\hline much & mæich & nosotros (nominativo) \\
\hline $\operatorname{mux}$ & aix & 1a plural (irregulares) \\
\hline mvch $\sim$ much & mæich & nosotros (genitivo) \\
\hline namnum & namnæm & caer (forma no finita) \\
\hline$n i c \sim n q u$ & $n i c \sim n q u$ & en (locativo) \\
\hline$\tilde{n} o f$ & nof & nosotros (objeto) \\
\hline$o c$ & $O C$ & nombre (poseído) \\
\hline
\end{tabular}




\begin{tabular}{|l|l|l|}
\hline pissi & pissi & mal (genitivo) \\
\hline piycan piycam & piycan & dar \\
\hline poleng & polæng & voluntad (poseído) \\
\hline tocum & tocæn & dejar \\
\hline vzi & xizi & tierra (genitivo) \\
\hline $\begin{array}{l}\text { xllangmuseyo } \\
\text { xllangmuse }\end{array}$ & $\begin{array}{l}\text { xllãgmusseio } \\
\text { xllangmusse }\end{array}$ & enemigo (genitivo) \\
\hline xllon & xllon & comida (poseído) \\
\hline yneng ynengo & ineng inengô & día \\
\hline yxllis & ixllæss & pecado (poseído) \\
\hline yzpuçère & mællæczære & habladuría \\
\hline
\end{tabular}

\section{Lo que contiene el Pater Noster}

Este pequeño texto tan sólo se encuentra en el Arte de Fernando de la Carrera. Consiste en una explicación del número de cosas que se pide en el Padre Nuestro y a quien se destinan las mismas. El texto en cuestión es el siguiente:

nite leck-ø-o tem-çæ-c e, mo Padre nuestro-ng_nic siete/cabeza-PS-EU/pedir-NOM־PS/ 3aS/ DET/padre nuestro-G/LOC

Siete pedidos hay en el Padre Nuestro

La frase nominal ñite lecнo temçæc ocupa el primer constituyente sintáctico. Nótese que el numeral ñite 'siete' no hace concordancia con el núcleo nominal temçæc que permanece en singular. Empero, lo que más llama la atención es la presencia del nombre lecнo 'cabeza', en su forma poseída. Sabemos que, por una metáfora, dicha palabra se utilizaba como unidad de las enumeraciones ordenadas y le atribuimos la glosa de 'primero', en tanto posición dentro de la sucesión, pero no como cantidad. A su vez, al analizar su distribución, notamos que se usa también para designar cantidades que tienen un orden. Por ejemplo, para los diez mandamientos: $\mathrm{Na}$ ssop lecнo (Carrera 1644: 164) o para los artículos de la fe que son catorce: $\mathrm{Na}$ ssop lecu allô nopæto (Carrera 1644: 213). ¿Por qué decimos que se trata de cantidades con un 
orden? Simplemente, porque se emplea con numerales que hacen referencia a conjuntos ordenados fijamente. No es igual el primer mandamiento que el quinto. Cada uno tiene su orden, dentro de una cantidad fija. No existe el undécimo mandamiento. Sin embargo, véase más adelante cómo hay un orden que determina para quién van los primeros pedidos y para quien los últimos. Pese a que no se emplean los ordinales, sí existe referencia a un conjunto ordenado que explicaría el uso de lecu. No obstante, esta particularidad léxica no se maneja en español, razón por la cual no se traduce. El segundo constituyente lo ocupa la flexión verbal $e$, a la que le hemos dado una traducción existencial, aun cuando también hubiésemos podido recurrir al sentido copulativo. Termina la oración una frase con el nombre de la oración que es tomado como préstamo y se le declina con el genitivo no poseído <ng>, que era propio de los lexemas acabados en vocal. El texto continúa con la determinación de quién se beneficia de los primeros pedidos:

çopæt-ô lecu-ø-o çio fe Dios-ø-i_iopæn

tres-EU/ primero-EU/ este/ COP/ Dios-ᄀPS-G/DAT

Esos tres primeros son para Dios.

Nuevamente, una frase con numerales da comienzo a esta oración. Aquí, lec $\mu$ funciona como una unidad que traducimos como 'primer(os)'. Los numerales cuando no cuantifican, directamente, un nombre precisan de un pronombre que haga las veces de núcleo de la frase nominal. En este caso, el pronombre es çio. El segundo constituyente lo ocupa la flexión $f e$ y luego el destinatario del pedido. Consecuentemente, la última frase establece el beneficiario de los pedidos finales:

nopæt-o quitzhi-e-io çio fe mæich peñ-æxs_pæn.

cuatro-EU/ último-PS-G-EU/ esto/ COP/ 1aPL-G/ bien-N-PS/DAT Esos cuatro del último son para nuestro bien.

Otra frase encabezada por un numeral nos confirma que, cuando no hay un nombre, se precisa de un pronombre (çio) como núcleo de la frase. Lo más destacado de esta oración es notar cómo los adjetivos 
podían comportarse, haciendo las veces de nombres en mochica. El adjetivo quitzhi 'último' recibe flexión de caso genitivo poseído, cual si fuese un nombre. Asimismo, el adjetivo peñ 'bueno' también recibe marca de posesión y declinación, al igual que una categoría nominal.

\section{Interlocución sobre el Pater Noster}

En el Arte de 1644, el Padre Nuestro viene acompañado de una interlocución que es un conjunto de nueve preguntas con sendas respuestas sobre lo que versa la plegaria en cuestión. El diálogo tiene fines pedagógicos, pues busca absolver aquellos puntos que debieron ser entendidos por los hablantes de mochica. He aquí la primera pregunta:

P. Ech_ix tem-apæc-o, çio tem-çæ-r-ær_nic.

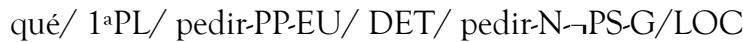

¿Qué (estamos) pidiendo en esta súplica?

La interlocución comienza con una palabra de pregunta ech en el primer constituyente, lo cual supone que es seguida por la flexión $i x$, que es algo que, en efecto, ocurre. Creemos que la flexión tiene tal forma y no la canónica, porque se cliticiza a la palabra de pregunta que termina en una consonante palatal. Sigue el verbo 'pedir' en su forma de participio presente o activo temapæco. Este participio se puede comportar como tema verbal y recibir desinencias (Carrera 1664: 140-141). De acuerdo con Middendorf (1892: 92), la acción de verbo en esta construcción tendría un aspecto progresivo o reiterativo. El nombre temçær de la última frase lo hemos traducido como 'súplica'. Nótese que la raíz de este nombre derivado de verbo es la misma que la del verbo principal.

R. Tem-apc-o_ix echech Dios-ø-i lec çiu-ng-ø mæich çio pij-c-æmnæm

pedir-PA-EU-1aPL/cosa-PL/Dios-PS-G/donde/3aS-G$\mathrm{AG} / 1^{\mathrm{aPL}} / 3^{\mathrm{a} S} /$ dar-CO-PAS-FIN

Estamos pidiendo el (reino) de Dios de (todas las) cosas, para sernos dado eso por él.

En la respuesta, el verbo temapco ocupa el primer constituyente, 
entonces, su flexión va pospuesta. La forma particular de la flexión que pierde su primera vocal nos hace pensar en que es un clítico de la raíz verbal, de ahí que ocupe otro constituyente sintáctico. Luego viene aquello que se pide, mediante una frase posposicional echech Diosi lec que requiere explicación. Según De la Carrera (1644: 130), la posposición lec puede traducir como "a casa de". En ese sentido, es similar a las preposiciones chez del francés o bei del alemán. Por tratarse de la divinidad, hemos cambiado "a casa de" por "al reino de". La expresión ech ech remite a las cosas y da una idea de abundancia y poder.

El verbo pijcæmnæm es pasivo, no finito y expresa finalidad. Este verbo al término de la frase rige todos los demás elementos a su izquierda. Reconocemos en él la raíz pij 'dar'. Este verbo tiene un agente y dos objetos. Por estar en pasiva, uno de los objetos pasa a ser sujeto y el sujeto de la activa puede aparecer como agente de manera opcional. Los verbos no finitos no asignan caso, así que sería lo esperado que el sujeto de la pasiva desaparezca; pero, como en la frase hay tres pronombres, asumimos que cada uno de ellos corresponde a los argumentos del verbo, de acuerdo con su valencia ${ }^{4}$. Así, el pronombre çio debe ser el sujeto de la pasiva (objeto inanimado de la activa). El pronombre genitivo çiung debe corresponder al agente opcional de la pasiva (sujeto de la activa), el cual no tiene una marca explícita, aun cuando cumple con tal rol. El pronombre mæich 'nosotros' debe ser el objeto que recibe lo que se da. De esta manera, çio es lo que se da, çiung es quien concede y mæich es a quien se otorga. Aquello que se da y es expresado con çio tiene correferencia con el reino de la divinidad de la cláusula anterior. El pedido continúa en los siguientes términos:

aie-çæn eix tem-apc-o çio Padre nuestro-ng_nic, mæich ixll-æss efco-çær-r.

y/1aPL/pedir-PA-EU/DET/padre nuestro-G/LOC/1aPL-G/

$4 \quad$ En las lenguas del mundo hay ejemplos de sujetos con verbos no finitos, como en ciertas construcciones de portugués: Eu trabalhava ali antes de eles chegarem, que literalmente dice: Yo trabajaba allí antes de ellos llegar. Es más, en algún caso, los sujetos pospuestos de los infinitivos pueden funcionar en español: Antes que viajar yo, prefieres venir tú. 
pecado-PS/perdonar-NOM־PS

asimismo estamos pidiendo en este Padre Nuestro, el perdón a nuestros pecados.

Así pues, la respuesta a esta primera pregunta se da a través de una oración compuesta, cuya segunda parte inicia con la conjunción aieçæn, en el primer constituyente. Por tanto, la flexión eix debe ir en el segundo constituyente, antecediendo al verbo temapco. Lo más interesante viene al final de la frase. Nos encontramos con un nombre deverbal efcoçær 'perdón', el cual tiene como objeto a la frase mæich ixllæss "nuestros pecados". Lo curioso es que esta última frase no está en genitivo y tal parece que o bien el nombre derivado de verbo efcoçær tenía la capacidad de asignar caso como si fuese un verbo, o bien se puso por error un nombre deverbal, en vez de un verbo no finito. De estas alternativas, nos inclinamos por la primera, por lo que veremos en la respuesta a la sexta pregunta. He aquí la segunda interrogante:

P. Ech_ix tem-apec-o, Dios-ø-i oc-ø mucha-ø ap lic-ssæc? qué/1aPL/pedir/PA/EU/Dios־PS-G/nombre-PS/adoración-PS/ pues/hacer-GER

¿Qué estamos pidiendo, pues, haciendo adoración al nombre de Dios?

La segunda pregunta tiene la estructura canónica de la oración mochica. En el primer constituyente está la palabra de pregunta ech. Consecuentemente, la flexión ix va en el segundo y después el verbo temapeco, que nuevamente tiene un tema participial. El verbo principal gobierna una cláusula que tiene su núcleo al final de la frase en la forma verbal no finita del gerundio licssæc 'haciendo'. Este gerundio rige los demás elementos a su izquierda, destacando la reaparición de la partícula ilativa ap 'pues', que estaba presente en la versión del Padre Nuestro de 1607 y ausente en la de 1644.

R. Tem-apc-o_ix santo-io çiu-ng oc-ø izc-ær-ø xam-ar-næm, aie-çæn izc-ær-ø irrim-c-æm-næm.

pedir-PA-EU-1aPL/santo-EU/3aS-G/nombre-PS/todo-7PS-G-AG/ reconocer-PAS-FIN/y/todo-PS-G-AG/temer-CAUS-PAS-FIN 
Estamos pidiendo (para) ser reconocido santo su nombre por todos, asimismo por todos (para) ser temido.

Si el tema verbal va en primera posición, su flexión va pospuesta. Luego vienen dos cláusulas coordinadas por la conjunción aieçæn. Cada una de las cláusulas tiene al final un verbo no finito que las gobierna. La primera cláusula es regida por el verbo pasivo xamarnæm. El morfema de pasiva está en armonía vocálica con la raíz del verbo. En esta pasiva, lo que más llama la atención es el agente, al cual le hemos asignado un morfema $ø$, pues, morfológicamente, sólo manifiesta el caso genitivo. La hipótesis que manejamos es que el caso del agente de pasiva $-n$, únicamente, se manifestaba con el genitivo de posesión -e, porque si se hubiese sufijado a los genitivos ng o ær, habría formado un haz de dos consonantes en la misma sílaba: ${ }^{*} n g n$ o ${ }^{*} æ r n$, lo cual era imposible en mochica. De ahí que no se haya realizado fónicamente, aun cuando sí formaba parte de la estructura de la frase. En la segunda cláusula, gobernada por el verbo pasivo irrimcæmnæm, también se encuentra la misma instancia de agente de pasiva. El morfema no finito -næm de ambas cláusulas expresaba finalidad, pero en algunos casos se comportaba como el germen de un infinitivo, sin esa carga semántica de una finalidad para el verbo. En esta traducción, funcionan ambas interpretaciones, así que hemos puesto entre paréntesis las preposiciones 'para' de finalidad. La tercera interrogante es la siguiente:

P. Ech_ix tem-apc-o, pij-c-an ñof tzhæng cuçia-s, çie eng-læc.

qué/ 1aPL/ pedir-PA-Eu/ dar-CO-IMP/ 1aPL-OBJ/ 2aS-G/ cieloPS/ si/ decir-GER

¿Qué estamos pidiendo si decimos, danos tu cielo?

La tercera pregunta tiene la estructura canónica de flexión en segunda posición que venimos comentando. Lo que más llama nuestra atención son las otras dos cláusulas. Al final de toda la oración hay un gerundio englæc 'diciendo' y tiene al lado una conjunción condicional çie 'si'. En la traducción, hemos tenido que adaptar esta estructura, porque en castellano el gerundio no funciona, en todos los casos, junto con el condicional. La oración "¿qué estamos pidiendo si diciendo, danos tu 
cielo?" no nos parece correcta. Por eso, como el verbo principal y el gerundio tienen un mismo agente, hemos traducido el texto con la misma primera persona plural. La cláusula a la izquierda de la anterior cláusula condicional es una cita literal de la plegaria original. Como esta cláusula es independiente, se da el caso que la raíz verbal pij 'dar' se encuentra en la primera posición, entonces, la flexión de imperativo an va pospuesta. Como respuesta se obtiene:

R. Tem-apæc-o_ix, Dios-ø mæich_nic chi-næm, santo-io çiu-ng chiçæ-r_er, aie-çæn eix tem-apc-o muc çæn, çiu-ng cuçia-s.

pedir-PA-EU-1aPL/ Dios $\neg$ PS/ 1aPL-G/ LOC /ser-FIN /santo-EU /3aSG/ser-NOM-PS/INS/y/ 1aPL/ pedir-PA-EU/allá/ y/3aS-G/cielo-PS

Estamos pidiendo (para) ser/estar Dios en nosotros, con su santa voluntad, asimismo, estamos pidiendo además allá, su cielo.

La tercera respuesta comienza por el verbo temapæco, lo cual supone que la flexión ix va pospuesta y su vocal $e$ cae para formar un clítico con la o final del primer constituyente. Sigue una cláusula con verbo no finito chinæm '(para) ser' al final de la misma. En esta frase, el sentido del verbo copulativo chi puede ser traducido como 'ser' o como 'estar'. Nos inclinamos, sin embargo, por 'ser', porque el verbo copulativo con el aspecto de 'estar' lo ostentaba la raíz loc (Carrera 1644: 126). La frase posposicional santoio çiung chiçær er 'con su santa voluntad' forma un paréntesis, porque está antes de la conjunción aieçæn y después del final de frase marcado por el verbo no finito chinæm, pero su sentido va con ambos verbos principales, pese a estar fuera de la cláusula no finita y no formar parte del conteo para la posición de la flexión del segundo verbo. Como quiera que fuere, la conjunción aieçæn ocupa el primer constituyente de la siguiente oración y, por tanto, la flexión eix antecede al verbo temapco. Lo que más interesa en esta segunda frase es la jerarquía que establecen las conjunciones. En efecto, la conjunción aieçæn coordina las dos oraciones principales, que están regidas por los participios temap(x)co. Por su parte, la conjunción çæn vincula el deíctico muc 'allá', con la frase nominal çiung cuçias 'su cielo'. La relación, en este caso, no es de coordinación; pues muc y çiung cuçias tienen la misma referencia. Más bien, la partícula çæn expresa una adición a los pedidos. No solamente se pide el ser de Dios, 
sino el lugar donde él mora. Como cuarta interrogante tenemos:

P. Ech_ix tem-apc-o ame eng-læc ei-æp-mang, tzhæng pol-æng_ mæn, mo æiz-ø-i_capæc, cuçia-ø-ng_nic_mæn?

Qué/1aPL/pedir/PA-EU/así/decir-GER/hacer-PAS-SUBJ-3a/2a, S-G/voluntad-PS/como/ DET tierra-PS-G/sobre/ cielo-ᄀPS/ LOC/como

¿Qué estamos pidiendo así diciendo: hágase según tu voluntad, sobre la tierra como en el cielo?

La cuarta pregunta está compuesta por tres cláusulas. La primera está formada por la palabra de pregunta ech 'qué' con el verbo principal temapco, cuya flexión lo antecede en segunda posición. La segunda cláusula la integran el adverbio ame 'así' y el gerundio englæc 'diciendo', que es un verbo no finito y, por ello, va al final de la frase. Este gerundio da paso a la tercera cláusula, que es una cita literal de la correspondiente oración del Padre Nuestro en mochica. El adverbio ame 'así' anticipa esta cita literal como una suerte de catáfora. La respuesta a esta cuestión es la siguiente:

R. Tem-apc-o_ix, izçæc ñofn-ø-ær-ø Dios-ø-i ssap-ø macu-qu-emnæm can ang cuçia-ø-ng_nic-o angel çio_mæn.

pedir-PA-EU-1aPL/ todo/ hombre-aPS-G-AG/ Dios-PS-G/ mandato-PS/ cumplir-CAUS-PAS-FIN/ REL/3 ${ }^{\text {s }}$ /cielo $\neg P S-G /$ LOC-EU/ángel/3aS/como

Estamos pidiendo ser cumplido el mandato de Dios por todo hombre, que está el ángel en el cielo, como ello.

La respuesta cuarta también posee tres cláusulas. La primera formada por el verbo principal en primera posición y su flexión pospuesta en segunda. Luego hay una cláusula que finaliza en el verbo no finito macuquemnæm, el cual está en voz pasiva. Nuevamente nos encontramos con un caso de verbo no finito en el que el sujeto está presente, pues la frase Diosi ssap 'mandato de Dios' es el sujeto. Esto lo explicamos en la nota cuarta. El agente de la pasiva también es explícito, ya que se manifiesta en la frase izçæc ñofnær 'por todo hombre'. La falta 
de marca en el agente y el uso del genitivo, en vez de él se explican por la hipótesis acerca de la formación de sílabas. Finalmente, viene una oración de relativo. Se cumple con que la conjunción ocupe el primer constituyente y la flexión el segundo. Hemos traducido, literalmente, la oración de relativo: "que está el ángel en el cielo, como ello", para poder mostrar la función de cada elemento léxico del mochica. Si hiciésemos una traducción que se adecuara más al español estándar, habríamos podido traducir la oración así: "tal como está el ángel en el cielo". Como siguiente interrogante se consigna:

P. Ech_ix tem-apxc-o, ame eng-læc aio ineng ineng-ôo mæich xllon-ø piy-c-an ñof alló mo lun-ø?

Qué/1aPL/pedir/PA-EU/así/decir-GER/DET/día/día-EU/1aPL-G/ comida-PS/dar-CO-IMP/ 1aPL-OBJ/CONJ/ DET/ día

¿Qué estamos pidiendo así diciendo: aquella nuestra comida del día a día danos también este día?

La quinta pregunta, nuevamente, se divide en tres cláusulas, con una estructura similar a la anterior cuestión. La primera cláusula la forman la palabra de pregunta ech, la flexión ix y el verbo principal temapæco, en ese orden. La segunda la conforman el adverbio ame, como catáfora del contenido de la tercera cláusula y el verbo no finito englæc, al término de dicha segunda cláusula. La tercera cláusula es una cita de la oración del Padre Nuestro en lengua mochica. Como contestación, se obtiene:

R. Tem-apæc-o_ix xllon-quic, mæich ærqu-eng-e_iopæn: aie-çæn peñ-o chi-çæ-c mæich moix-ø-e_iopæn.

pedir-PA-EU-1aPL/comida-רPS/1aPL-G/cuerpo-PS-G/DAT/y/ buen-EU/ser-NOM-ᄀPS/1aPL-G/alma-PS-G/DAT

Estamos pidiendo la comida para nuestro cuerpo, asimismo bienestar para nuestra alma.

La respuesta quinta empieza por la raíz verbal temapæco y, por tanto, pospone la flexión ix que se adapta a la vocal $o$. Enseguida, vienen los atributos que se piden y para quien se destinan los mismos. Así, la comida que se designa con la voz xllonquic es para nuestro cuerpo y, por 
ello, se usa la frase mæich ærquengeiopæn. La conjunción aieçæn coordina ambos atributos. De esta manera, es empleada la frase peño chiçæc que, literalmente, se traduce como 'ser bueno' en tanto bienestar para nuestra alma, mediante la frase mæich moixeiopæn. El diálogo continúa con la siguiente consulta:

P. Ech_ix tem-apc-o, ame eng-læc ef-qu-ec-an ñof ixll-æss, aie, acan ax ef-co xllangmu-ss-e-io, mæich, çio_mæn?

qué/1 ${ }^{\mathrm{aPL}} /$ pedir/PA-EU/así/decir-GER/ perdonar-CAUS-CO$\mathrm{IMP} / 1^{\mathrm{aPL}} \mathrm{OB} \mathrm{BJ} /$ pecado-PS

así como/1aPL/perdonar-CAUS/enemigo-PS-G-EU/1ªL-OBJ/ eso/como

¿Qué estamos pidiendo así diciendo: perdónanos nuestros pecados, así como perdonamos nosotros de nuestros enemigos, como eso?

La sexta pregunta sigue el modelo de las anteriores. Tres cláusulas: Una principal con palabra de pregunta, flexión y verbo, una intermedia con adverbio y gerundio, y una final con la cita del pasaje correspondiente de la oración en cuestión. Sobre lo que queremos llamar la atención es que la flexión se presenta como ax, en vez de aix, que es la manera como aparece en el pasaje, de donde la cita fue tomada. Esto nos confirma, en primer lugar, que el timbre de la vocal en la flexión -lejos de ser un error tipográfico- experimentaba, efectivamente, un caso de asimilación. En segundo lugar, la ausencia de $l a\langle i\rangle$ es un indicio del carácter, meramente, fonético de este segmento, el cual estaba determinado por un entorno palatal. Se absuelve la demanda en los siguientes términos:

R. Tem-apc-o_ix, mæich, ixll-æss ef-co-çæ-r aie-çæn eix tem-apæc-o purgatorio-ng_niqu_ich-ò tuxllum-çæx-r.

pedir-PA-EU-1 aPL/1 aPL-G/pecado-PS/perdonar-NOM-PS/ y/1aPL/pedir-PA-EU/purgatorio-G/LOC/AB-EU/salir-NOM-PS

Estamos pidiendo el perdonar nuestros pecados, asimismo estamos pidiendo la salida del purgatorio.

La sexta respuesta coordina dos oraciones que poseen el mismo verbo principal. En la primera oración simple la flexión $i x$ va pospuesta 
como clítico, porque el primer constituyente lo ocupa el tema participial temapco. En la segunda, la flexión eix antecede al verbo, porque la primera posición la ocupa la conjunción aieçæn. Los objetos directos de ambos verbos principales son nombres deverbales. Así, la primera oración repite la estructura de la primera pregunta, en la que el deverbal efcoçær vuelve a regir la frase nominal mæich ixllæss "nuestros pecados". Esto sería un indicio razonable de que el nombre efcoçær podía asignar caso y regir al objeto mæich ixllæss. Por su parte, el núcleo del objeto directo de la segunda oración simple también era un nombre deverbal tuxllumçær 'salida'. Este nombre rige una frase posposicional integrada por la marca de límite de constituyente $o$, la cual indica que todo lo que sigue es complemento, el ablativo <ich>, el locativo <niqu>, el genitivo $\langle n g\rangle$ y el préstamo purgatorio. La interpelación da paso a la siguiente frase:

P. Exp_ix eng-apc-o ame aie acan ax ef-co xllangmu-ss-e-io, mæich, çio_mæn?

porqué/1aPL/decir-PA-EU/así/asícomo/1aPL/perdonar/enemigoPS-G-EU/1aPL/3aS/como

¿(Por) qué estamos diciendo así: tal como nosotros perdonamos del enemigo, como eso?

La séptima interrogante varía en relación con las anteriores. Lo que es un misterio es la palabra de pregunta $\langle E x p\rangle$ que no aparece más que una vez en la edición de 1644 . Ahora bien, sabemos por las reglas de silabificación del mochica que el encuentro vocálico $\langle e x\rangle$ no existe. La palabra de pregunta que más se le parece es epæn 'por qué' (Carrera 1644: 124), que según el lingüista noruego Hovdhaugen (2004: 16) es la contracción de ep 'porqué' + pæn 'por'. En efecto, existe la palabra de pregunta ep 'por qué' (Carrera 1644: 152), que sería una segunda opción. De otro lado, la edición del Arte de 1880 (Carrera 1880: 103) trae como palabra de pregunta ech 'qué', en el pasaje correspondiente. Tres son, entonces, las posibilidades. Pese a que la $p$ de ep no es palatal, hemos encontrado ejemplos de ep junto con la flexión ix (Carrera 1644: 247), así que por el lado de la flexión es difícil hacer inferencias. Por el lado de la respuesta, tanto la pregunta con 'qué' como la que se formula con 'por qué' tienen sentido. Por ello, en la traducción, hemos puesto entre 
paréntesis la preposición por. Ante esta aporía sólo nos queda señalar el error y establecer las posibles soluciones a la espera de más datos. La interrogante se resuelve así:

R. Ino_ng ef-co_ca Dios-ø mæich ixll-æss çie mam-ix ef-co ænta, chang-cæed-e-io mæich.

NEG/3aS/perdonar/FUT/Dios־PS/1aPL/pecado-PS/si/SUBJ1aPL/perdonar/NEG/prójimo-PS-G-EU/1aPL

No perdonará Dios nuestros pecados, si no perdonásemos nosotros del prójimo.

La respuesta séptima comienza con una negación 〈ino〉. De momento, es difícil establecer si la negación era tan sólo $\langle$ in $\rangle$ y la $o$ correspondía a una marca de límite. Lo que sí sabemos es que la flexión $\langle n g\rangle$ en segunda posición se le adjunta como clítico. Esto nos permite determinar que la partícula ca era un adverbio temporal que permitía construir el futuro, pero no formaba parte del sistema flexivo y, por ello, no ocupaba el segundo constituyente. El verbo principal efco rige dos objetos: Dios y mæich ixllæss "nuestros pecados". La segunda oración es encabezada por la conjunción condicional çie 'si' en primer constituyente. Le sigue la flexión de subjuntivo mamix en el segundo constituyente. Esta flexión es distinta a los ejemplos que proporciona De la Carrera (1644: 40, 50, 57 y 71), en los que la flexión es tan sólo maix. Ahora bien, lejos de un error tipográfico, creemos que esta desviación del patrón normal refleja una estrategia para romper el encuentro de vocales con la inserción de una consonante. Como el primer constituyente lo ocupa la conjunción y el segundo la flexión, el sujeto mæich 'nosotros' se halla al final de la oración. Este sujeto rige la frase en genitivo changcædeio "del prójimo". Tal construcción, en la que el genitivo está determinado permite la ausencia de un núcleo que haga referencia a los 'pecados'. Una traducción más cercana al castellano sería: "No perdonará Dios nuestros pecados, si no perdonásemos nosotros los del prójimo”. Sin embargo, hemos preferido mantenernos lo más cercano posible a lo literal. La penúltima interrogante de este cuestionario es:

P. ech_ix tem-apc-o ame eng-læc am_oz tocæn ñof xllangmu-ss-e mællæc-çæ-re_nic nam-næm? 
qué/1aPL/pedir/PA-EU/asi/decir-GER/NEG/2S/dejar/1aPL-OBJ/ enemigo-PS-G/hablar-NOM-PS-G/LOC/caer-FIN

¿Qué estamos pidiendo así diciendo: no nos dejes caer en las habladurías del enemigo?

La penúltima pregunta vuelve a la estructura ya conocida de tres cláusulas con una palabra de pregunta y un verbo que subcategoriza una forma verbal no finita y ésta da paso a una cita del Padre Nuestro. Su respuesta es como sigue:

R. Tem-apc-o_ix Dios-ø-i_lec tarr-o chi-çæ-r, a-çæn ixll-ø-i_nic namssæc, ssap-ø_er, lock-ø_er-çæn ænta-çæn çifa chi-çæ-r_er. pedir-PA-EU-1aPL/Dios-PS-G/ con/fuerte-EU/ser-NOM-PS/ no-y/pecado-PS-G/LOC/caer-GER

Estamos pidiendo el ser fuerte con Dios y no caer en pecado, con la boca y con el ojo tampoco con el propio ser

La contestación penúltima tiene detalles interesantes, destacando el carácter negativo de la conjunción açæn. Si se tradujese la conjunción sin el sentido negativo implícito, la oración carecería de lógica. Otra particularidad es que hay una cláusula con un verbo no finito namssæc 'caer' y después de él vienen una serie de frases posposicionales unidas por al menos una conjunción negativa ${ }^{5}$. La demanda final de este diálogo es la siguiente:

P. Eñ-o piss-ø-i_nqu_ich-o_ix eng ef-quem-næm, ame eng-læc lecpna_n ef-co ñof piss-ø-i_nqu_ich?

5 Habíamos dicho en la nota dos que, al igual que el alemán, el mochica posiciona sus formas no finitas al final de la frase. La explicación a estos complementos después del verbo no finito la encontramos en la gramática alemana, a través del concepto de Nachfeld o 'campo posterior'. Cuando las oraciones poseen mucha información, se pueden poner los complementos después del verbo no finito o de los participios. Esto se aplica por ejemplo, a atributos preposicionales. La frase "Hast du heute ein Kind mit einer roten Hose gesehen?" (literalmente: ¿Has tú hoy un niño con un rojo pantalón visto?) puede tener el complemento preposicional después del participio, al final de la frase: Hast du heute ein Kind gesehen mit einer roten Hose? (literalmente: ¿Has tú hoy un niño visto con un rojo pantalón?). 
Quién-G/mal-PS-G/LOC/AB-EU/1aPL/querer/librar-PAS-FIN/ así/decir-GER/másbien/IMPlibrar/1ªLOBJ/mal-PS-G/LOC/AB ¿Del mal de quién queremos ser librados, así diciendo: más bien líbranos del mal?

La última interrogante también se aparta del patrón de preguntas. Comienza con una frase posposicional, que tiene una marca $o$ de límite de constituyente. Esto nos prueba que tal marca no es un adjetivador como se viene diciendo. Siguen tres casos, ablativo <ich〉, locativo $\langle n q u>$ y genitivo $\langle i\rangle$, que nominaliza el adjetivo piss 'mal'. La partícula <eño $\rangle$ es una forma genitiva de la palabra de pregunta correspondiente a 'quién'. La flexión ix se une como clítico a este primer constituyente, pero manteniéndose en la segunda posición. Luego viene una construcción modal, el verbo eng 'querer' rige una forma pasiva no finita efquemnæm, en la que se pierde por completo el sentido de finalidad del morfema -næm, actuando plenamente como un infinitivo. Vienen luego la cláusula de gerundio ame englæc "así diciendo", en la que ame 'así' funciona como catáfora y la cita correspondiente a la oración del Padre Nuestro en mochica. He aquí la absolución de la pregunta:

R. Demonio-ng mæck-ø-qu_ich-ô_ix tem ef-quem-næm, aie-cæn infierno-ø-ng_niqu_ich, aie-çæn tim-æn-o piss-ø-i_nqu_ich.

Demonio-G/mano-PS-G/AB-EU/1aPL/querer/librar-PAS-FIN/y/ infierno-PS-G/LOC/AB/

y/otro-PL-EU/mal-G/LOC/AB

De la mano del demonio queremos ser librados, asimismo del infierno, y de otros males.

La respuesta final comienza con una frase posposicional en ablativo ich. Esta posposición rige a la forma $\langle m x c \mu q u\rangle$, que corresponde a la contracción de mæcнæc (Carrera 1644: 161), con la caída de la vocal $\langle x\rangle$. La contracción mæcuæc es la fusión de la forma no poseída mæcra (Carrera 1644: 6) y el locativo nic (Carrera 1644: 159). Sabemos que se trata de la forma poseída, porque la forma no poseída está documentada como mæcuquic 'mano' (Carrera 1644: 5). Pues bien, la forma mæcuqu rige, a su vez, al préstamo demonio que está declinado en caso genitivo. 
Al final de la frase posposicional se encuentra la ô que marca el límite de tal constituyente. Así, al ocupar la primera posición, la flexión verbal (e) $i x$ antecede a la raíz verbal y se adjunta como clítico a este constituyente perdiendo su vocal inicial, al fusionarla con la ô del primer constituyente. Pospuesta a la flexión se encuentra la raíz verbal tem 'querer', que rige la forma pasiva no finita efquemnæm '(para) ser libre'. Nótese cómo el sentido de finalidad está ausente en esta construcción y, más bien, el morfema -næm se comporta como un verdadero infinitivo. Tras la forma no finita, están dos frases posposicionales antecedidas por sendas conjunciones que, antes que coordinar, cumplen el papel de adicionar. Estos sintagmas también ocuparían la posición de Nachfeld que hemos mencionado en la nota quinta. Es interesante ver cómo el plural -æn, en la última frase nominal se halla en el determinante tim 'otro', en vez del núcleo piss 'mal'.

\section{Conclusiones}

Como conclusión de este trabajo, ofrecemos las traducciones de los textos que hemos analizado. Siguiendo el orden que establecimos en la traducción, pondremos la versión de Jerónimo de Oré, luego la de Fernando de la Carrera. Después vienen aquellos textos que, exclusivamente, realizó este último, en donde se establece lo que contiene el Pater Noster y la interlocución sobre esa oración.

\section{Pater Noster (Oré)}

Nuestro padre que estás en el cielo. Hágase pues adoración a tu nombre. Danos tu cielo. Hágase según tu voluntad sobre la tierra como en el cielo. Aquella nuestra comida del día a día, danos también este día. Perdónanos nuestros pecados, como perdonamos nosotros de nuestros enemigos, como eso. No nos dejes caer en las habladurías de nuestros enemigos. Más bien, líbranos del mal. 


\section{Pater Noster (Carrera)}

Nuestro padre, que estás en el cielo. Hágase adoración a tu nombre. Danos tu cielo. Hágase según tu voluntad sobre la tierra como en el cielo. Aquella nuestra comida del día a día danos también este día. Perdónanos nuestros pecados, así como perdonamos nosotros de nuestros enemigos, como eso. No nos dejes caer en las habladurías de nuestros enemigos. Más bien, líbranos del mal.

\section{Lo que contiene el Pater Noster}

Siete pedidos hay en el Padre Nuestro. Esos tres primeros son para Dios. Esos cuatro del último son para nuestro bien.

\section{Interlocución sobre el Pater Noster}

¿Qué estamos pidiendo en esta súplica?

Estamos pidiendo el reino de Dios de todas las cosas, para sernos dado eso por él, asimismo estamos pidiendo en este Padre Nuestro, el perdón a nuestros pecados.

¿Qué estamos pidiendo, pues, haciendo adoración al nombre de Dios? Estamos pidiendo ser reconocido santo su nombre por todos, asimismo por todos ser temido.

¿Qué estamos pidiendo si decimos danos tu cielo?

Estamos pidiendo (para) ser Dios en nosotros, con su santa voluntad, asimismo, estamos pidiendo además allá, su cielo.

¿Qué estamos pidiendo así diciendo: hágase según tu voluntad, sobre la tierra como en el cielo?

Estamos pidiendo ser cumplido el mandato de Dios por todo hombre, que está el ángel en el cielo, como ello.

¿Qué estamos pidiendo así diciendo: aquella nuestra comida del día a día, danos también este día? 
Estamos pidiendo la comida para nuestro cuerpo, asimismo bienestar para nuestra alma.

¿Qué estamos pidiendo así diciendo: perdónanos nuestros pecados, así como perdonamos nosotros de nuestros enemigos, como eso?

Estamos pidiendo perdonar nuestros pecados, asimismo estamos pidiendo salida del purgatorio.

¿(Por) qué estamos diciendo así: tal como nosotros perdonamos del enemigo, como eso?

No perdonará Dios nuestros pecados, si no perdonásemos nosotros del prójimo.

¿Qué estamos pidiendo así diciendo: no nos dejes caer en las habladurías del enemigo?

Estamos pidiendo el ser fuerte con Dios y no caer en pecado, con la boca y con el ojo tampoco con el propio ser

¿Del mal de quién queremos ser librados, así diciendo: más bien líbranos del mal?

De la mano del demonio queremos ser librados, asimismo del infierno, asimismo de otros males. 


\section{BIBLIOGRAFÍA}

ADELAAR, Willem F. H. \& Pieter Cornelis Muysken. The Languages of the Andes. Cambridge: Cambridge University Press, 2004.

ADELUNG, Johann Christoph. Mithridates oder allgemeine Sprachenkunde. Berlin: Vossische Buchhandlung, dritter Teil, zweite Abteilung, 1813.

BRÜNING, Hans Heinrich. Mochica Wörterbuch (= Patrimonios: Lingüistica II). Lima: USMP, [1905-1924] 2004.

CARRERA DAZA, Fernando de la. ARTE DE LA LENGVA YVNGA DE LOS VALLES del Obispado de Truxillo del Peru, con un Confessonario, y todas las Oraciones Christianas, traducidas en la lengua, y otras cosas. Lima, Ioseph de Contreras, 1644.

- Arte de la lengua yunga del Obispado de Trujillo del Perú. Edición de Carlos Paz Soldán. Lima, Imprenta Liberal, [1644] 1880.

GRASSERIE, Raoul de la. "Langue Yunga (dialecte mochica). Textes inédites trouvés à la Bibliothèque Nationale de Paris." En Actas de la Undécima Reunión del Congreso Internacional de Americanistas en México, 1895. México, Agencia Tipográfica de F. Díaz de León. Tomo I, pp. 499 -503, [1895] 1897.

HERVÁS Y PANDURO, Lorenzo. Saggio Pratico delle lingue (= Idea dell'Universo, tomo XXI). Cesena, Gregorio Biasini all'Insegna di Pallade, 1787.

HOVDHAUGEN, Even. Mochica (= Languages of the World/Materials 433). Muenchen, LINCOM GmbH, 2004. 
. “How Was Mochica Being Pronounced?" En ZWARTJES, Otto y Cristina Altman (eds.): Missionary Linguistics II / Lingüistica Misionera II. Orthography and Phonology. Selected papers from the Second International Conference on Missionary Linguistics, São Paulo, 10-13 March 2004 (= Series III . Studies in the History of the Language Sciences, volume 109). Amsterdam/Philadelphia: John Benjamins Publishing Company, pp.171-180, 2005.

MIDDENDORF, Ernst Wilhelm. Das Muchik oder die Chimu-Sprache (= Die einheimischen Sprachen Perus, sechster Band). Leipzig, F. A. Brockhaus, 1892.

ORÉ, Luis Jerónimo de (= Lvdovicum Hieronymvm Orerium). RITVALE, SEV MANVALE PERVANVM, et forma brevis administrandi apud Indos sacrosancta Baptismi, Ponitentiæ, Eucharistiæ, Matrimonij, \& Extremæ vnctionis Sacramenta. Neapoli, Apud Io. Iacobum Carlinum \& Constantinum Vitales, 1607.

SALAS GARCÍA, José Antonio. "La fórmula para el sacramento de la comunión en la lengua mochica”. Allpanchis, número 70, pp. 73-98, 2007.

.Formas ligadas en los numerales del mochica". Lexis, volumen XXXII, número 1, pp. 147-158, 2008a.

.La Señal de la Cruz en la lengua mochica". Lexis, volumen XXXII, número 2, pp. 321-345, 2008b.

.Los sentidos corporales en mochica". Boletín de la Academia Peruana de la Lengua, volumen 48, pp. 75-89, 2009a.

.Los enemigos del alma en lengua mochica". Escritura y Pensamiento, número 25, pp. 23-35, $2009 \mathrm{~b}$. 
TERCER CONCILIO LIMENSE. DOCTRINA CHRISTIANA, Y CATECISMO PARA INSTRVCCION de los Indios, y de las de mas personas, que han de ser enseñadas en nuestra sancta Fé. CON VN CONFESSIONARIO, Y OTRAS COSAS necessarias para los que doctrinan. Edición facsimilar de la de Antonio Ricardo. Lima, Ediciones Copé de Petroperú, [1584] 1984.

TORERO FERNÁNDEZ DE CÓRDOVA, Alfredo. Idiomas de los Andes. Lingüistica e historia (= Travaux de l'Institut Français d'Études Andines 162). Lima, IFEA \& Editorial Horizonte, 2002.

ZEVALLOS QUIÑONES, Jorge. “Toponimia preincaica en el norte del Perú". Cuadernos de estudios del Instituto de Investigaciones Históricas de la Universidad Católica del Perú, tomo II, número 5, pp. 205-247, 1943.

\section{Correspondencia:}

José Antonio Salas García

Licenciado en Lingüística y Literatura Hispánica con mención en Lingüística por la Pontificia Universidad Católica del Perú.

Correo electrónico: jos778@hotmail.com 